\title{
On the problem of low-temperature ordering in ferro-antiferroelectric mixed compounds
}

\author{
N.A.Korynevskii ${ }^{1,2}$, V.B.Solovyan ${ }^{1}$ \\ ${ }^{1}$ Institute for Condensed Matter Physics of the National Academy of Sciences of Ukraine, \\ 1 Svientsitskii Str., 79011 Lviv, Ukraine \\ 2 Institute of Physics, University of Szczecin, 15 Wielkopolska Str., 70451 Szczecin, Poland
}

Received March 6, 2009, in final form May 18, 2009

\begin{abstract}
The problem of microscopic description of low-temperature phases in ferro-antiferroelectric mixed compounds is discussed. Those phases (ferro-, antiferroelectric and dipole glass) are interpreted based on the specific distributions for single and pair correlation functions in a non-ergodic thermodynamic system. Phase diagram and dielectric susceptibility of a mixed system are obtained and analyzed.
\end{abstract}

Key words: ferroelectrics, solid mixtures, phase diagram, dipole glass

PACS: $75.10 . H k, 77.22 . C h, 77.84 . F$

The recently introduced and analysed model $[1,2]$ for microscopic investigation of ferroelectricantiferroelectric mixed compounds with hydrogen bonds predicts numerous solutions for single and pair correlation functions of interactive particles. The single correlation functions are directly connected with an order parameter (ferro- and antiferroelectric), but pair correlation functions describe a possible dipole glass phase and in its origin they are close to a well-known Edwards and Anderson spin glass parameter [3]. In the limit of small $(n \leqslant 0,45)$ and large $(n \geqslant 0,85)$ concentrations of ferroelectric component, the investigated system possesses antiferroelectric and ferroelectric properties up to the very low temperatures, respectively. However, for intermediate concentration $(0,45<n<0,85)$ there arises a specific ordered phase with only short range correlations between particles. The number of different types of correlations (the number of different solutions for pair correlation functions) increase with temperature falling down [4]. Each of these pair correlation functions describe some specific arrangement of the nearest neighbours in disordered ferro-antiferroelectric systems. Since such systems are usually prepared due to high-speed cooling the distribution of such correlation functions is quite random. So, in order to calculate thermodynamic functions, the averaging procedure should be performed not for a partition function, as in Gibbs system, but for its logarithm, directly connected with the observed quantities.

Our approach differs from the well-known methods of investigating the mixed KDP-type compounds (see [5-8]). Those methods are based on the random fields taken into account, that are induced by competition between ferro- and antiferroelectric types of interaction in hydrogen bonds lattice. In this way, the dynamical properties of a mixed system were investigated too [9]. We have proposed a model of randomly distributed interacting groups $\left(\mathrm{Rb}-\mathrm{PO}_{4}\right.$ and $\left.\mathrm{NH}_{4}-\mathrm{PO}_{4}\right)$ which form a regular crystalline lattice. So, the microscopic proton-proton interactions are not considered directly. Such an approach essentially simplifies the calculations and provides good physical results.

For rubidium ammonium dihydrogen phosphate $-\mathrm{Rb}_{n}\left(\mathrm{NH}_{4}\right)_{1-n} \mathrm{H}_{2} \mathrm{PO}_{4}$ (RADP) and isomorphous rubidium ammonium dihydrogen arsenate - $\mathrm{Rb}_{n}\left(\mathrm{NH}_{4}\right)_{1-n} \mathrm{H}_{2} \mathrm{AsO}_{4}$ (RADA) mixed compounds, this scheme of calculations (using replica method [10]) has shown the existence of at least two areas of different non-trivial solutions for pair correlation functions [4]. Namely, a high temperature paraelectric phase with only one type of pair correlation function; the second one: dipole glass phase $G_{1}$ with three types of them; the third one: dipole glass phase $G_{2}$ with five types of them. In every phase, one type of solution is "trivial", so it exists for any temperature, including only the 
temperature of paraelectric phase. The aim of the present paper is a more precise investigation of our model in order to find new dipole glass phases and to analyse the behaviour of thermodynamic functions in a large scale of temperatures and concentrations.

We regard a regular crystalline lattice of KDP-type system, each site of which is occupied (quite randomly) by rubidium or ammonium groups, that is by $\mathrm{Rb}$ or $\mathrm{NH}_{4}$. The dipole momenta of $\mathrm{Rb}-$ $\mathrm{AsO}_{4}$ group are directed along $z$-axis of the crystal and the dipole momenta of $\mathrm{NH}_{4}-\mathrm{AsO}_{4}$ group are directed along the $x$-axis. In the first case we can observe a ferroelectric ordering (below certain temperature) and in the second case the antiferroelectric ordering is possible. Due to competition between ferroelectric and antiferroelectric types of ordering, the crystal undergoes frustration: no type of ordering is preferential and instead of long range order only short range correlations between particles remain. At low temperature and at intermediate concentration, those correlations form a specific state of a crystal, i. e., a dipole glass phase.

The Hamiltonian of ferroelectric-antiferroelectric mixed system is as follows $[1,2]$ :

$$
H=-\frac{1}{2} \sum_{i, j} \sum_{m, m^{\prime}}\left\{V_{i j}^{m m^{\prime}} \hat{n_{i}} \hat{n_{j}} S_{i m}^{z} S_{j m^{\prime}}^{z}+U_{i j}^{m m^{\prime}}\left(1-\hat{n_{i}}\right)\left(1-\hat{n_{j}}\right) S_{i m}^{x} S_{j m^{\prime}}^{x}\right\}
$$

Here $V_{i j}^{m m^{\prime}}$ is the intensity of interaction between $m$-th "dipole particle" $\mathrm{Rb}-\mathrm{AsO}_{4}$ in $i$-th site with $m^{\prime}$-th "dipole particles" $\mathrm{Rb}-\mathrm{AsO}_{4}$ in $j$-th site and $U_{i j}^{m m^{\prime}}$ is the same for $\mathrm{NH}_{4}-\mathrm{AsO}_{4}$ "dipole particles". So, the first term in (1) is responsible for ferroelectric ordering and the second term is responsible for antiferroelectric ordering in the mixed system. $S^{x}, S^{z}$ are projections of the unit classical vector $\vec{S}$ on the $x$ and the $z$ axis, respectively; the site occupation operators $\hat{n}_{i}$ have the following eigenvalues:

$$
n_{i}=\left\{\begin{array}{cc}
1, & \text { when } i \text {-th site is occupied by } \mathrm{Rb} \\
0, & \text { when } i \text {-th site is occupied by } \mathrm{NH}_{4}
\end{array}\right.
$$

Since the unit cell for KDP-type crystal contains two formula units (there are also two sublattices below a phase transition point [11]), the numbers $m, m^{\prime}$ run from 1 to 2 . So, we can use the two-particle cluster approximation in which the interaction of particles belonging to the same site is taken exactly, but for particles belonging to different sites the self-consistent field [4] is sufficient. Another great problem connected with the calculation of thermodynamic functions is configuration averaging over randomly distributed "dipole particles" ( $\mathrm{Rb}-\mathrm{AsO}_{4}$ and $\left.\mathrm{NH}_{4}-\mathrm{AsO}_{4}\right)$ with $z$-and $x$-oriented dipole momenta all over the sites of the crystalline lattice. This problem can be solved using the replica method [4,10]. Since we have two sets of variables $\left\{S_{i m}^{z}\right\}$ and $\left\{S_{i m}^{x}\right\}$, the introduction of $n$ replica variables $\sigma_{i m}^{k}$ for $S_{i m}^{z}$ and $n$ replica variables $\xi_{i m}^{k}$ for $S_{i m}^{x}$ is necessary.

With the accuracy up to the second order of symmetric replica expansion (variables $\sigma_{i m}^{k}$ and $\xi_{i m}^{k}$ reduce to $\left.\sigma_{i m}, \xi_{i m}\right)$ and using the two-particle cluster approximation, the following expansion for $-\beta H$ has been obtained [12]:

$$
-\beta H=\sum_{i}\left\{A_{1} \sigma_{i 1} \sigma_{i 2}+B_{1}\left(\sigma_{i 1}+\sigma_{i 2}\right)+A_{2} \xi_{i 1} \xi_{i 2}+B_{2}\left(\xi_{i 1}-\xi_{i 2}\right)\right\}-C,
$$

where $A, B, C$ are some coefficients connected with initial parameters $V_{i j}^{m n^{\prime}}, U_{i j}^{m m^{\prime}}$ of the Hamiltonian and "order" parameters $P, q, g_{z}, g_{x}$ :

$$
\begin{aligned}
& A_{1}=J_{1}+J_{2}\left[\frac{V_{\mathrm{f}}^{2}-V_{\mathrm{af}}^{2}}{V^{2}}+\left(1+\left(\frac{V_{\mathrm{f}}-V_{\mathrm{af}}}{V}\right)^{2}\right) g_{z}-\frac{U}{V} g_{x}\right], \\
& A_{2}=\left(\tanh \beta V-J_{1}\right) \frac{U}{V}+J_{2}\left[\frac{U_{\mathrm{f}}^{2}-U_{\mathrm{af}}^{2}}{V^{2}}+\left(\left(\frac{U}{V}\right)^{2}+\left(\frac{U_{\mathrm{f}}-U_{\mathrm{af}}}{V}\right)^{2}\right) g_{x}-\frac{U}{V} g_{z}\right],
\end{aligned}
$$




$$
\begin{aligned}
& B_{1}=J_{1} \frac{V_{\mathrm{f}}}{V} P+4 J_{2}\left[\left(\frac{V_{\mathrm{f}}}{V}\right)^{2} P^{3}-\frac{V_{\mathrm{f}}}{V} \frac{U_{\mathrm{af}}}{V} P q^{2}\right], \\
& B_{2}=\left(\tanh \beta V-J_{1}\right) \frac{U_{\mathrm{af}}}{V} q+4 J_{2}\left[\left(\frac{U_{\mathrm{af}}}{V}\right)^{2} q^{3}-\frac{V_{\mathrm{f}}}{V} \frac{U_{\mathrm{af}}}{V} P^{2} q\right] \text {, } \\
& C=\left\{J_{1} \frac{V_{\mathrm{f}}}{V} P^{2}+\left(\tanh \beta V-J_{1}\right) \frac{U_{\mathrm{af}}}{V} q^{2}+\frac{J_{2}}{2}\left[-2+\left(g_{z}-\frac{U}{V} g_{x}\right)^{2}\right.\right. \\
& \left.\left.+\left(\frac{V_{\mathrm{f}}+V_{\mathrm{af}}}{V}\right)^{2}\left(1+g_{z}\right)^{2}+\left(\frac{U_{\mathrm{f}}+U_{\mathrm{af}}}{V}\right)^{2}\left(1+g_{x}\right)^{2}+24\left(\frac{V_{\mathrm{f}}}{V} P^{2}-\frac{U_{\mathrm{af}}}{V} q^{2}\right)^{2}\right]\right\} ; \\
& V_{\mathrm{f}}=\frac{1}{2} \sum_{j=1}^{N}\left(V_{i j}^{11}+V_{i j}^{12}\right), \quad V_{\mathrm{af}}=\frac{1}{2} \sum_{j=1}^{N}\left(V_{i j}^{11}-V_{i j}^{12}\right), \\
& U_{\mathrm{f}}=\frac{1}{2} \sum_{j=1}^{N}\left(U_{i j}^{11}+U_{i j}^{12}\right), \quad U_{\mathrm{af}}=\frac{1}{2} \sum_{j=1}^{N}\left(U_{i j}^{11}-U_{i j}^{12}\right), \\
& V=V_{i i}^{12}, \quad U=U_{i i}^{12} \text {. } \\
& J_{1}=\sum_{t=1}^{\infty}(-1)^{t} 2 \ln \left(1-n+n \mathrm{e}^{-2 \beta V t}\right) \\
& J_{2}=\sum_{t=1}^{\infty}(-1)^{t} 4 t \ln \left(1-n+n \mathrm{e}^{-2 \beta V t}\right) ; \\
& P=\overline{\left\langle\sigma_{i 1}\right\rangle}, \quad q=\overline{\left\langle\xi_{i 1}\right\rangle}, \quad g_{z}=\overline{\left\langle\sigma_{i 1} \sigma_{i 2}\right\rangle}, \quad g_{x}=\overline{\left\langle\xi_{i 1} \xi_{i 2}\right\rangle} ;
\end{aligned}
$$

$\beta=\frac{1}{k T}, k$ is the Boltzman constant, $T$ is the absolute temperature. Here $\langle\ldots\rangle$ denotes thermal averaging for a given distribution of $z$ - and $x$-oriented dipole momenta of $\mathrm{Rb}-\mathrm{AsO}_{4}$ and $\mathrm{NH}_{4}-\mathrm{AsO}_{4}$ groups, respectively, whereas a bar denotes a stochastic averaging over different distributions. We shall use the random distribution of $\mathrm{Rb}$ and $\mathrm{NH}_{4}$ groups all over the crystal. $P$ and $q$ are simple ferroelectric and antiferroelectric order parameters, respectively. $g_{z}$ and $g_{x}$ are the nearest neighbours pair correlation functions (like Edwards and Anderson spin glass parameter).

Performing the trace operation in the expression for free energy

$$
F=-\frac{1}{\beta} \ln \operatorname{Tr}\left\{\mathrm{e}^{-\beta H}\right\}
$$

over cluster states of spin-like variables $\sigma$ and $\xi$, and configuration averaging using an effective version of replica method [10], the final formula for $F$ has been obtained:

$$
F=-\frac{1}{\beta} \ln \left\{4\left(\mathrm{e}^{-A_{1}}+\mathrm{e}^{A_{1}} \cosh 2 B_{1}\right)\left(\mathrm{e}^{A_{2}}+\mathrm{e}^{-A_{2}} \cosh 2 B_{2}\right)\right\}+\frac{1}{\beta} C .
$$

Minimization procedure of (7) under all the introduced "order" parameters $P, q, g_{z}, g_{x}$ gives a set of equations for their determination:

$$
\begin{aligned}
P & =Z^{-1} \mathrm{e}^{A_{1}} \sinh 2 B_{1}\left(\mathrm{e}^{A_{2}}+\mathrm{e}^{-A_{2}} \cosh 2 B_{2}\right), \\
q & =Z^{-1} \mathrm{e}^{-A_{2}} \sinh 2 B_{2}\left(\mathrm{e}^{-A_{1}}+\mathrm{e}^{A_{1}} \cosh 2 B_{1}\right), \\
g_{z} & =Z^{-1}\left(\mathrm{e}^{-A_{1}}-\mathrm{e}^{A_{1}} \cosh 2 B_{1}\right)\left(\mathrm{e}^{A_{2}}+\mathrm{e}^{-A_{2}} \cosh 2 B_{2}\right), \\
g_{x} & =Z^{-1}\left(\mathrm{e}^{-A_{1}}+\mathrm{e}^{A_{1}} \cosh 2 B_{1}\right)\left(\mathrm{e}^{A_{2}}-\mathrm{e}^{-A_{2}} \cosh 2 B_{2}\right), \\
Z & =\left(\mathrm{e}^{-A_{1}}+\mathrm{e}^{A_{1}} \cosh 2 B_{1}\right)\left(\mathrm{e}^{A_{2}}+\mathrm{e}^{-A_{2}} \cosh 2 B_{2}\right) .
\end{aligned}
$$

The solutions of (8) may be found by numerical calculations only, because all parameters $A_{1}, A_{2}, B_{1}, B_{2}$ are strongly non-linear functions of temperature and concentration. The basic solutions of (8) have 
been obtained in [2], where one area for the existence of simulations of three different roots for $g_{z}, g_{x}$ was found, and in [4], where an additional area for five different roots for $g_{z}, g_{x}$ was detected. So, it was shown that dipole glass phase is characterized by a set of different roots for pair correlation functions of the nearest neighbours.

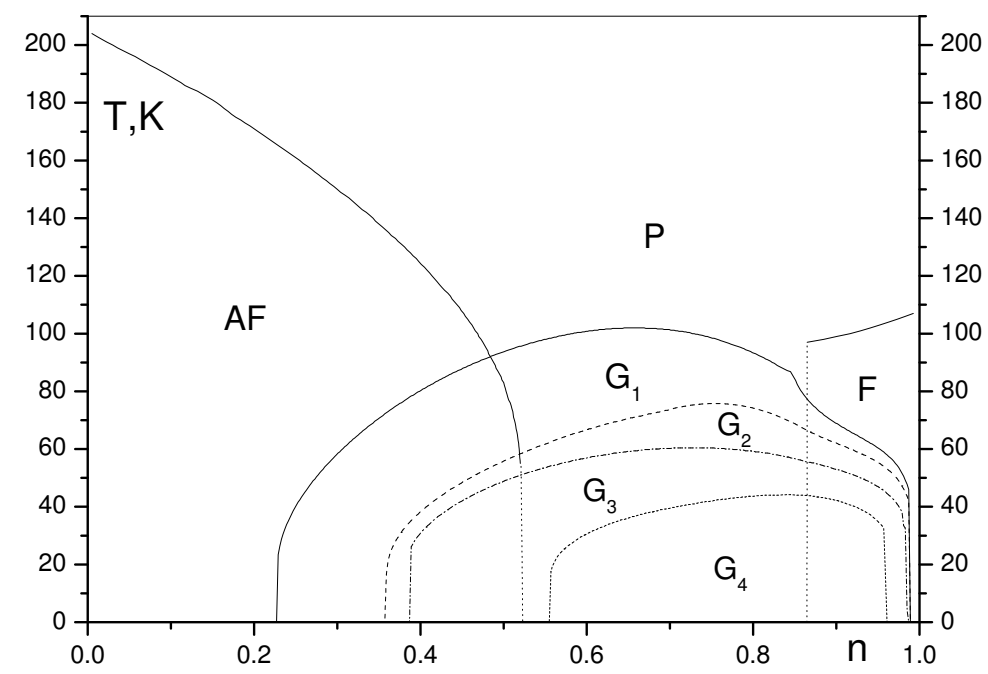

Figure 1. Phase diagram of RADA type compound built for the model Hamiltonian parameters: $V=65 K, U=-210 K, V_{\mathrm{f}}=70 K, U_{\mathrm{f}}=-40 K, V_{\mathrm{af}}=-80 K, U_{\mathrm{af}}=120 K$.

Using a highly precise method for numerical solution of a set of non-linear equations (8) we have found five regions with different numbers of roots for $g_{z}, g_{x}$, starting with region $P$ (one root), and the next are: region $G_{1}$ (three roots), region $G_{2}$ (five roots), region $G_{3}$ (seven roots) and region $G_{4}$ (nine roots) (see figure 1). So, for different values of temperature and concentration (at sufficiently low temperature and for intermediate concentration) there is a set of local states in ferroelectric-antiferroelectric mixed system. The lower is the temperature, the more complicated is the dipole glass phase (the bigger number of different pair correlation functions is realized).

Comparing figure 1 to the classical experimental results for RADA [11,13,14] one may find that regions $G_{2}, G_{3}, G_{4}$ completely coincide with the dipole glass phase observed experimentally. However, the region $G_{1}$ lies in sufficiently higher temperature range. What's the matter? In our opinion the region $G_{1}$ (three roots for $g_{z}, g_{x}$ are realized) present the phase of precursor dipole glass state. Three different types of interparticle correlations show only a slight deviation of pure paraelectric phase ( $P$ in figure 1 ) from homogenous state. But in regions $G_{2}-G_{4}$ such deviations are substantial, the distribution of interparticle correlations in them is sufficiently non-ergodic. They really present the dipole glass phase. The possibility of precursor of dipole glass phase existence was claimed in $[15,16]$, where the polar nano-clusters which appear at rather high temperatures and remain to exist down to low temperatures were detected using Raman scattering method. The recent investigations [17,18] also confirm the existence of precursor dipole glass phase at temperatures about $100-120 \mathrm{~K}$ in mixed RADA materials.

It is important to note the coexistence of ferroelectric-dipole glass $(F-G)$ and antiferroelectricdipole glass $(A F-G)$ phases (see figure 1), which take place practically in the whole region of concentration for ferroelectric phase, but only in rather small region of concentration for antiferroelectric phase. This fact is in good agreement with experiment $[11,14,17]$.

In order to perform a more detailed investigation of different ordered and disordered phases of RADA in a wide region of temperatures and concentrations the analysis of basic thermodynamic functions is essential. In the dipole glass phase, the free energy (7) possesses a set of local minima according to different solutions of equations (8) [19]. Those minima are separated by potential barriers with non-limited height in the thermodynamic limit $N \rightarrow \infty$. Due to these potential barriers, the investigated mixed system remains near some minima for a sufficiently long time. So, we have a remarkable example of non-ergodic system, because the time averaging values do not coincide with 
the ensemble averaging values for them. In order to correctly calculate thermodynamic functions, e. g., the free energy, all potential minima should be taken into account. We shall use "nonuniform" mean values, because the states with lower energy contribute mostly to the observed quantities. For free energy we have

$$
F=\frac{\sum_{l=1}^{p} F_{i} \mathrm{e}^{-\beta F_{l}}}{\sum_{l=1}^{p} \mathrm{e}^{-\beta F_{l}}},
$$

where $F_{l}$ is the expression (7) for $l$-th solution of set of equations (8), $l$ run from 1 to $p, p$ being a number of different roots of (8) in separate regions $\left(P, F, A F, G_{k}\right.$, see figure 1$)$. For example, at concentration $n \approx 0,6$ and temperature $T \approx 50 \mathrm{~K} p=7$, because there are 7 roots in the region $G_{3}$.

Static dielectric susceptibility $\chi=\left(\frac{\partial P}{\partial E}\right)_{E=0}$ we shall calculate using the thermodynamic identity:

$$
\mathrm{d} F=-S \mathrm{~d} T-P \mathrm{~d} E
$$

where $E$ is an external electric field.

To obtain free energy depending on $E$, the term

$$
\frac{\beta}{2} \sum_{i}\left\{E\left(\sigma_{11}+\sigma_{21}\right)+E\left(\xi_{1 j}+\xi_{2 i}\right)\right\},
$$

describing the energy of the investigated system in the electric field, should be introduced into expression (2). As a result, for the "partial" free energy $F_{l}$, instead of the expression (7), a new one takes place:

$$
\begin{aligned}
F_{l}= & -\frac{1}{\beta} \ln \left\{4\left[\mathrm{e}^{-A_{1}^{(l)}}+\mathrm{e}^{A_{1}^{(l)}} \cosh \left(2 B_{1}^{(l)}+\beta E\right)\right]\right. \\
& {\left.\left[\mathrm{e}^{A_{2}^{(l)}} \cosh \beta E+\mathrm{e}^{-A_{2}^{(l)}} \cosh 2 B_{2}^{(l)}\right]\right\}+\frac{1}{\beta} C^{(l)} }
\end{aligned}
$$

Here $A_{1}^{(l)}, A_{2}^{(l)}, B_{1}^{(l)}, B_{2}^{(l)}, C^{(l)}$ are formulae (3) with $P^{(l)}, q^{(l)}, g_{x}^{(l)}, g_{z}^{(l)}$ parameters forming one root.

The general expression for $\chi$ now takes the form:

$$
\chi=\left.\frac{\sum_{l}\left[\frac{\partial P^{(l)}}{\partial E}+\beta\left(P^{(l)}-P\right) P^{(l)}\right] \mathrm{e}^{-\beta F_{l}}}{\sum_{l} \mathrm{e}^{-\beta F_{l}}}\right|_{E=0}
$$

where

$$
P=\frac{\sum_{l} P^{(l)} \mathrm{e}^{-\beta F_{l}}}{\sum_{l} \mathrm{e}^{-\beta F_{l}}} .
$$

For the regions of concentration and temperature, where long range ordering in mixed ferroantiferroelectric system does not exist, the expression (13) may be substituted by:

$$
\chi=-\frac{\sum_{l} \frac{\partial^{2} F_{l}}{\partial E^{2}} \mathrm{e}^{-\beta F_{l}}}{\sum_{l} \mathrm{e}^{-\beta F_{l}}}
$$

The temperature dependencies of inverse dielectric susceptibility (in arbitrary units) calculated based on (14) for some concentration $0,55 \leqslant n \leqslant 0,85$ are presented in figure 2 . According to figure 1 only paraelectric and pure dipole glass phases exist in this region. Moreover, the dipole glass phase is divided into several zones, from $G_{1}$ - precursor of dipole glass state to $G_{4}$ - the state with strongly non-ergodic distributions of interparticle correlations.

The behaviour of $1 / \chi$ in general is close to the law predicted by Curie, especially at low and high temperatures. However, for temperatures corresponding to transitions between different $G_{l}$ regions, there arise some perturbations in the linear behaviour of $1 / \chi$. Most peculiarities of $\chi$ take place when the precursor of dipole glass phase $\left(G_{1}\right)$ appears. At low temperatures, the appearance of a new dipole glass phase $\left(G_{4}\right.$ in figure 1 for example) insufficiently contribute into the dielectric susceptibility because the total non-ergodic character of the investigated system internal arrangement changes insignificantly in this case. 


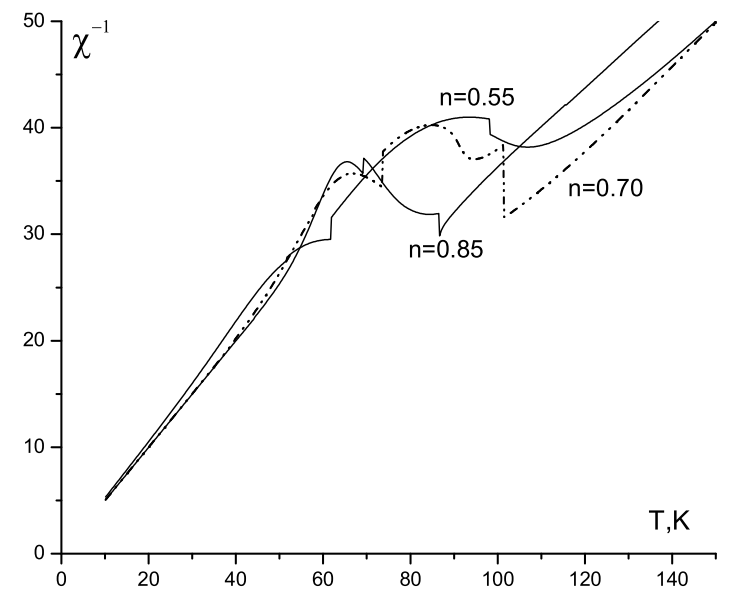

Figure 2. Inverse dielectric susceptibility of RADA type compound at different concentrations of $\mathrm{Rb}$ atoms. The model Hamiltonian parameters are the same as in figure 1.

\section{References}

1. Korynevskii N.A., Baran O.R., Ferroelectrics, 2004, 300, 151.

2. Korynevskii N.A., Solovyan V.B., Ferroelectrics, 2005, 317, 19.

3. Edwards S.F., Anderson P.W., J. Phys. E, 1975, 5, 965.

4. Korynevskii N.A., Solovyan V.B., Phase Transitions, 2007, 80, 55.

5. Matsushita E., Matsubara T., Prog. Theor. Phys., 1984, 71, 235.

6. Matsushita E., Matsubara T., J. Phys. Soc. Jap., 1985, 54, 1161.

7. Pirc R., Tadic B., Blinc R., Phys, Rev. B, 1987, 36, 8607.

8. Kutnjak Z., Pirc R., Levstik A., Levstik I., Filipic C., Blinc R., Phys. Rev. B, 1994, 50, 12421.

9. Levitskii R.R., Sorokov S.I., Stankowski J., Trybula Z., Vdovych A.S., Condens. Matter Phys., 2008, 11, 523 .

10. Bidaux R., Carton J.P., Sarma G., J. Phys. A: Math. Gen., 1976, 9, L87.

11. Trybula Z., J.Stankowski, Condens. Matt. Phys., 1998, 1, 311.

12. Korynevskii N.A., Solovyan V.B., Rev. Adv. Mater. Sci., 2006, 12, 90.

13. Kim S., Kwun S., Phys. Rev. B, 1990, 42, 638.

14. Trybula Z., Kaszynski J., Malustynska H., Ferroelectrics, 2005, 316, 125.

15. Yuzyuk Yu.I., Torgashev V.I., Farhit R., Gregova I., Petzelt J., Simon P., De Sousa Meneses D., Rabkin L.M., J. Phys.: Condens. Matter, 1998, 10, 1157.

16. Buixaderas E., Kamba S., Petzelt J., Ferroelectrics, 2004, 308, 131.

17. Trybula Z., Kaszynska K., Trybula M., Levitskii R.R. Book of Abstracts of IX Polish-Ukrainian Meeting and XXIX International School on Ferroelecrics Physics, p.24, Krakow, Poland, 2008.

18. Kamba S. (private communication).

19. Korynevskii N.A., Solovyan V.B., Condens. Matt. Phys., 2006, 9, 187. 


\title{
До питання про низькотемпературне впорядкування у сегнето-антисегнетоелектричних змішаних сполуках
}

\author{
М.А.Кориневський ${ }^{1,2}$, В.Б.Солов'ян ${ }^{1}$ \\ 1 Інститут фізики конденсованих систем НАН України, 79011 Львів, вул. Свєнціцького, 1 \\ 2 Інститут фізики щецінського університету, Польща, 70451 щецін, вул. Вєлькопольська, 15 \\ Отримано 6 березня 2009 р., в остаточному вигляді - 18 травня 2009 р.
}

Обговорюється питання мікроскопічного опису низькотемпературних фаз у сегнето-антисегнетоелектричних змішаних сполуках. Ці фази (сегнето-, антисегнетоелектрична і дипольного скла) інтерпретуються на основі специфічних розподілів для унарних і парних кореляційних функцій в неергодичних термодинамічних системах. Отримано і проаналізовано фазову діаграму та діелектричну сприйнятливість змішаної системи.

Ключові слова: сегнетоелектрики, тверді розчини, фазова діаграма, дипольне скло

PACS: 75.10.Hk, 77.22.Ch, 77.84.F 
